\title{
Haptic Feedback and Sensory Substitution during Telemanipulated Suturing
}

\author{
M. Tavakoli R.V. Patel M. Moallem \\ Canadian Surgical Technologies \& Advanced Robotics \\ 339 Windermere Road \\ Department of Electrical and Computer Engineering \\ University of Western Ontario, \\ London, Ontario N6A 5A5, Canada \\ London, Ontario N6A 5B9, Canada \\ E-mail:tavakoli@uwo.ca,rajni@eng.uwo.ca,mmoallem@engga.uwo.ca
}

\begin{abstract}
${ }^{*}$
Various modes of sensory feedback to the user have the potential to enhance performance in robot-assisted surgery. In this paper, it is hypothesized that substituting or augmenting force feedback by visual representation of the force levels can potentially assist the user in limiting the amount of applied forces. In addition to confirming the above for a telemanipulated suturing task, the results indicate that there is a trade-off between the magnitudes of applied forces and the time required to complete the task.
\end{abstract}

\section{Introduction}

An important problem with conventional endoscopic surgery concerns the loss or distortion of tactile, kinesthetic and force feedback from tissue and its interaction with the instrument [1]. Although this problem can be solved using teleoperated surgical robots, the currently available robotic systems for surgery (the da Vinci and the Zeus systems from Intuitive Surgical Inc.) do not yet address the problem of haptic feedback to the surgeon. This is while haptic feedback during robotic teleoperation has been shown to improve the performance of surgical task by reducing contact forces, the number of errors, and the task completion times [2]. It is interesting to see the effect of substitution or augmentation of haptic feedback by a visual representation of haptic information on the user's performance.

A bar indicator whose height varies with the magnitude of grip forces has been added to a version of the Zeus system, thus providing visual feedback of tool/tissue interactions to the user. While such a "sensory substitution" (also called "visual force feedback") can be a short-term solution to the problems caused by lack of haptic feedback, it requires constant visual attention and may be tiring. It is generally believed that using a force-reflective user's console provides a more efficient and intuitive means of relaying haptic information to the surgeon.

\footnotetext{
*This research was supported by the Ontario Research and Development Challenge Fund under grant 00-May-0709, infrastructure grants from the Canada Foundation for Innovation awarded to the London Health Sciences Centre (Canadian Surgical Technologies and Advanced Robotics) and the University of Western Ontario, the Natural Sciences and Engineering Research Council (NSERC) of Canada under grants RGPIN-1345 and RGPIN-227612, and the Institute for Robotics and Intelligent Systems of the Precarn, Inc. under the CSA-IRIS grant.
}

Quantifying the difference in the user's performance between manual operation and robotic teleoperation of suturing in presence of (auditory/visual) sensory substitution has been the subject of a recent study [3]. Nevertheless, it remains to be seen what the difference in terms of performance is between sensory substitution and actual haptic feedback during robotic teleoperation. As such, using the magnitudes of applied forces and the task completion times as the performance metrics, in this paper we compare the suturing performance when (a) haptic feedback is provided to the user's hand, and (b) haptic feedback is substituted or augmented by visual information.

\section{System Description}

A master-slave system appropriate for use in an endoscopic surgery environment has been developed [1]. Through the master interface, a user controls the motion of the slave arm (surgical tool) and receives force/torque feedback of slave-environment interactions while the environment is shown to the user via 2-D camera vision. The master and slave subsystems, tailored for establishing force-reflective teleoperation only in the twist direction (i.e. rotations about the instrument axis) are shown in Figure 1a. To pierce the tissue, an arced suturing needle (Ethicon PG-J346 size 0) is bent near the blunt end and just in front of where it is held by the slave instrument, such that the needle moves in a circular arc as the instrument rotates about its axis. In Figure 1a, the sixteen lightemitting diodes located near the monitor and used to form the bar indicator for visual force feedback are also shown.

\section{Experiment Design}

Seven subjects (4 males, 3 females) aged 24-34 participated in the suturing experiments on 3 different tissue samples all made of foam material but with stiffness increasing from material \#1 to material \#3. Subjects had little to average exposure to haptic feedback, no exposure to visual sensory substitution, and no experience with suturing. Each test consisted of 10 trials (i.e., 10 suturing operations of the needle). In each trial of each test and for each tissue, the contact forces between the instrument and the tissue were recorded for subsequent analysis. Four different tests were conducted in which, in addition to the camera vision, subjects received various forms of sensory feedback about the interaction between the instrument and the tissue. 
In Test 1 , the subjects received visual force feedback (VFF) with a low feedback gain. In Test 2 , the subjects received (actual) force feedback (FF) with a low feedback gain. In Test 3, the subjects received force feedback (FF) with a high feedback gain. In Test 4 , the subjects received both visual force feedback and force feedback (VFF+FF) with a high feedback gain.

\section{Results and Discussion}

Based on the experiments, for the situations in which haptic feedback is replaced or augmented by visual force feedback, performance comparisons were made in terms of (a) the magnitude of instrument-tissue interactions (when the needle is pushed in during each suturing operation of the needle), and (b) the time to complete each test. In the graphs that follow, $\tau_{z}$ is the torque about the instrument axis due to the instrument-tissue interaction with positive and negative values corresponding to the cases where the needle is pushed into and pulled out of tissue, respectively.

Conclusion 1: Comparing the results of Tests 1 and 2, it was found that VFF can reduce the peak and average contact forces as compared to FF, provided the users pay attention to the visual representation of force levels. In some cases, where it took users longer to complete the task under VFF (as they had to constantly refer to the visual display to see and limit the forces), the forces exerted on the tissue were lower compared to FF (e.g., subject \#7 with material \#1 as shown in Figure 1b). One reason for this is that the sensitivity of a visual force indicator is only limited by the resolution of the force measurements while the sensitivity of the human hand for force sensing is limited in nature $(0.5 \mathrm{~N}$ or $7 \%$ is the just-noticeable-difference [4]). In other cases, however, where the time to perform the task under VFF was less than FF, the applied forces turned out to be higher than FF. This shows that constant visual atten- tion to the visual indicator could cause fatigue and once a user did not pay enough attention to the presented visual information, he/she would compromise the magnitude of applied forces for the time to complete the task. This may be a major drawback to VFF especially for dexterous tasks, in which keeping track of several visual force indicators is more difficult and distracting. To summarize, our results showed that for a 1-DOF task on soft tissue and for a short period of time, VFF could provide sufficient feedback of an instrument's contact with tissue and outperform FF in terms of exerting less forces on the tissue.

Conclusion 2: Comparing the results of Tests 3 and 4, it was determined that supplying both VFF and FF at the same time could be better than providing FF alone. With $\mathrm{VFF}+\mathrm{FF}$, it normally took users longer than FF to complete the task, but in return the exerted forces were smaller (e.g. subject \#3 with material \#3 as shown in Figure 1c). In some cases, $\mathrm{VFF}+\mathrm{FF}$ even helped the users to apply forces more consistently after the tissue had been punctured, but at the expense of time.

\section{References}

[1] M. Tavakoli, R.V. Patel and M. Moallem, "Haptic Interaction in Robot-Assisted Endoscopic Surgery: A Sensorized End Effector," To appear in the Int. J. Medical Robotics \& Computer Assisted Surgery, Vol. 1, No. 2, 2005.

[2] C. Wagner, N. Stylopoulos, and R. Howe, "Force feedback in surgery: Analysis of blunt dissection," 10th Symp. Haptic Interfaces for Virtual Environment and Teleoperator Systems, Orlando, Florida, 2002.

[3] M. Kitagawa, D. Dokko, A.M. Okamura, B.T. Bethea, and D.D. Yuh, "Effect of Sensory Substitution on Suture Manipulation Forces for Surgical Teleoperation," Medicine Meets Virtual Reality, Newport Beach, California, 2004.

[4] K.B. Shimoga, "A survey of perceptual feedback issues in dextrous telemanipulation: Part I. Finger force feedback," IEEE Annual Virtual Reality International Symp., Seattle, Washington, 1993.
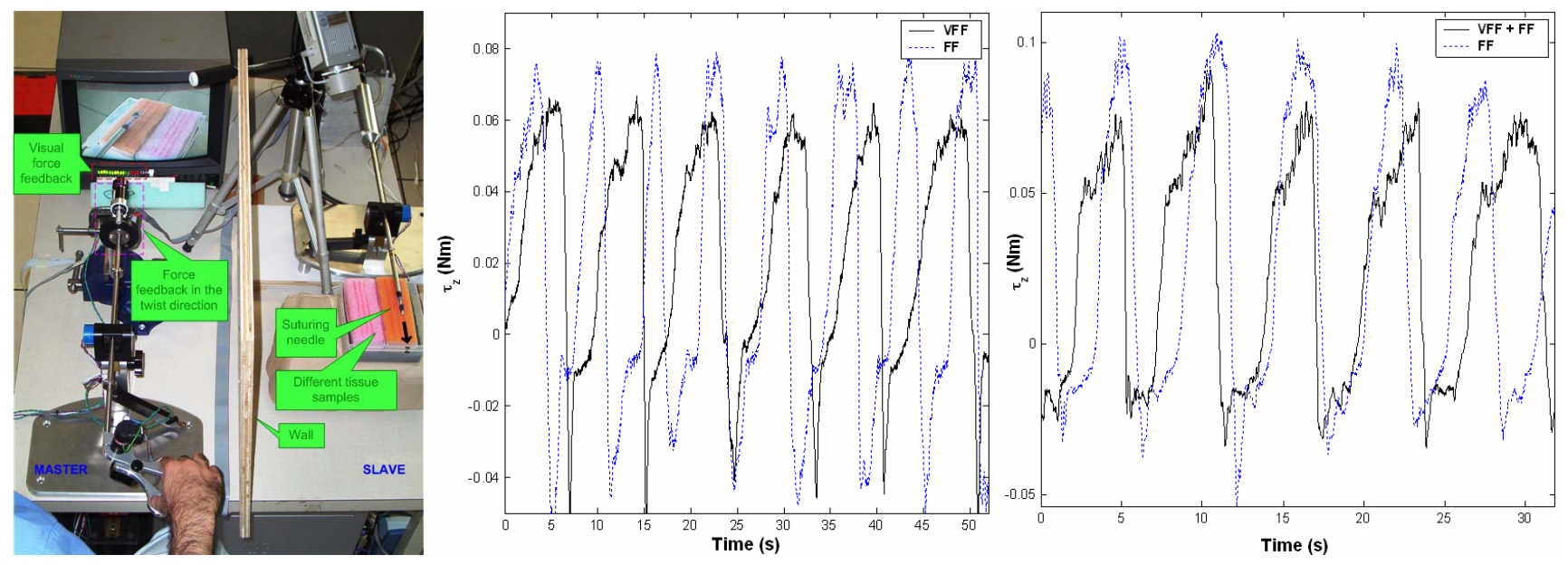

Figure 1: From left to right: (a) the master-slave setup for performing telemanipulated suturing tests, (b) measured torques with VFF and with FF, (c) measured torques with VFF+FF and with FF. 